\title{
SOFT CONNECTED PROPERTIES AND IRRESOLUTE SOFT FUNCTIONS BASED ON B-OPEN SOFT SETS
}

\author{
Alaa Mohamed Abd El-latif
}

\begin{abstract}
In this paper, we introduce another application on the b-open soft sets [10], by studying the notion of connectedness based on it. We give basic definitions and theorems about it. Further, we introduce the notion of b-irresolute soft functions as a generalization of the b-continuous soft functions and study their properties in detail. Finally, we show that the surjective b-irresolute soft image of soft b-connected space is also soft b-connected.

Keywords: Soft b-connected; Soft b-component; Soft b-hyperconnected; Soft b-separated; Soft b-connected; b-irresolute soft functions
\end{abstract}

\section{Introduction}

Theories such as theory of vague sets and theory of rough sets are considered as mathematical tools for dealing with uncertainties. But these theories have their own difficulties. The concept of soft sets was first introduced by Molodtsov [25] in 1999 as a general mathematical tool for dealing with uncertain objects in order to solve complicated problems in economics, engineering and other disciplines.

After presentation of the operations of soft sets [24], the properties and applications of soft set theory have been studied increasingly [7, 26, 27]. To develop soft set theory, the operations of the soft sets are redefined and a uni-int decision making method was constructed by using these new operations [9].

Recently, in 2011, Shabir and Naz [29] initiated the study of soft topological spaces. They defined soft topology on the collection $\tau$ of soft sets over $X$. Consequently, they defined basic notions of soft topological spaces such as open soft and closed soft sets, soft subspace, soft closure, soft nbd of a point, soft separation axioms, soft regular spaces and soft normal spaces and established their several properties. Hussain and Ahmad [13] investigated the properties of open (closed) soft, soft nbd and soft closure. They also defined and discussed the properties of soft interior,

Received April 14, 2016; accepted July 22, 2016

2010 Mathematics Subject Classification. Primary 54A05, 54A40; Secondary 54C08, 54D20 
soft exterior and soft boundary which are fundamental for further research on soft topology and will strengthen the foundations of the theory of soft topological spaces.

It got some stability only after the introduction of soft topology [29] in 2011. In [14], Kandil et al. introduced some soft operations such as semi-open soft, pre-open soft, $\alpha$-open soft and $\beta$-open soft and investigated their properties in detail. Kandil et al. [21] introduced the notion of soft semi-separation axioms. The notion of soft ideal was initiated for the first time by Kandil et al. [17]. They also introduced the concept of soft local function. These concepts are discussed with a view to find new soft topologies from the original one, called soft topological spaces with soft ideal $(X, \tau, E, \tilde{I})$. Applications to various fields were further investigated by Kandil et al.[15, 16, 18, 19, 20, 22]. The notion of supra soft topological spaces was initiated for the first time by El-sheikh and Abd El-latif [11], which is extended in [3]. The notion of b-open soft sets was initiated in $[6,10]$ and extended in [28]. An applications on b-open soft sets were introduced in $[1,12]$.

In this paper, we introduce and study the notion of connectedness based on the notion of b-open soft sets and give basic definitions and theorems about it. Further, we introduce the notion of b-irresolute soft functions as a generalization to the b-continuous soft function and study their properties in detail. Finally, we show that the surjective b-irresolute soft image of soft b-connected space is also soft b-connected.

\section{Preliminaries}

Definition 2.1. [25] Let $X$ be an initial universe and $E$ be a set of parameters. Let $P(X)$ denote the power set of $X$ and $A$ be a non-empty subset of $E$. A pair $(F, A)$ denoted by $F_{A}$ is called a soft set over $X$, where $F$ is a mapping given by $F: A \rightarrow P(X)$. In other words, a soft set over $X$ is a parameterized family of subsets of the universe $X$. For a particular $e \in A, F(e)$ may be considered the set of $e$-approximate elements of the soft set $(F, A)$ and if $e \notin A$, then $F(e)=\phi$ i.e $F_{A}=\{(e, F(e)): e \in A \subseteq E, F: A \rightarrow P(X)\}$. The family of all these soft sets denoted by $S S(X)_{A}$.

Definition 2.2. [29] Let $F_{E}$ be a soft set over $X$ and $x \in X$. We say that $x \in F_{E}$ read as $x$ belongs to the soft set $F_{E}$ whenever $x \in F(e)$ for all $e \in E$.

Definition 2.3. [29] Let $\tau$ be a collection of soft sets over a universe $X$ with a fixed set of parameters $E$, then $\tau \subseteq S S(X)_{E}$ is called a soft topology on $X$ if

(1) $\tilde{X}, \tilde{\phi} \in \tau$, where $\tilde{\phi}(e)=\phi$ and $\tilde{X}(e)=X, \forall e \in E$,

(2) the union of any number of soft sets in $\tau$ belongs to $\tau$,

(3) the intersection of any two soft sets in $\tau$ belongs to $\tau$. 
The triplet $(X, \tau, E)$ is called a soft topological space over $X$.

Definition 2.4. $[7,13]$ The complement of a soft set $F_{A}$, denoted by $F_{A}^{c}, F^{c}$ : $A \rightarrow P(X)$ is mapping given by $F^{c}(e)=X-F(e), \forall e \in A$ and $F^{c}$ is called the soft complement function of $F$. Also, $F_{A}$ is said to be closed soft set in $X$, if its relative complement $F_{A}^{c}$ is open soft set.

Definition 2.5. [13] Let $(X, \tau, E)$ be a soft topological space. The members of $\tau$ are said to be open soft sets in $X$. We denote the set of all open soft sets over $X$ by $O S(X, \tau, E)$, or when there can be no confusion by $O S(X)$ and the set of all closed soft sets by $C S(X, \tau, E)$, or $C S(X)$.

Definition 2.6. [29] Let $(X, \tau, E)$ be a soft topological space and $F_{A} \in S S(X)_{E}$. The soft closure of $F_{A}$, denoted by $\operatorname{cl}\left(F_{A}\right)$ is the intersection of all closed soft super sets of $F_{A}$. Clearly $\mathrm{cl}\left(F_{A}\right)$ is the smallest closed soft set over $X$ which contains $F_{A}$ i.e $c l\left(F_{A}\right)=\tilde{\cap}\left\{H_{C}: H_{C}\right.$ is closed soft set and $\left.\left.F_{A} \tilde{\subseteq} H_{C}\right\}\right)$.

Definition 2.7. [30] Let $(X, \tau, E)$ be a soft topological space and $F_{A} \in S S(X)_{E}$. The soft interior of $G_{B}$, denoted by $\operatorname{int}\left(G_{B}\right)$ is the union of all open soft subsets of $G_{B}$. Clearly $\operatorname{int}\left(G_{B}\right)$ is the largest open soft set over $X$ which contained in $G_{B}$ i.e $\operatorname{int}\left(G_{B}\right)=\tilde{\cup}\left\{H_{C}: H_{C}\right.$ is an open soft set and $\left.\left.H_{C} \tilde{\subseteq} G_{B}\right\}\right)$.

Definition 2.8. [30] The soft set $F_{E} \in S S(X)_{E}$ is called a soft point in $\tilde{X}$ if there exist $x \in X$ and $\alpha \in E$ such that $F(\alpha)=\{x\}$ and $F\left(\alpha^{c}\right)=\phi$ for each $\alpha^{c} \in E-\{\alpha\}$, and the soft point $F_{E}$ is denoted by $x_{\alpha}$.

Definition 2.9. [30] The soft point $x_{\alpha}$ is said to be belonging to the soft set $G_{A}$, denoted by $x_{\alpha} \tilde{\in} G_{A}$, if for the element $\alpha \in A, F(\alpha) \subseteq G(\alpha)$.

Definition 2.10. [29] Let $(X, \tau, E)$ be a soft topological space, $F_{E} \in S S(X)_{E}$ and $Y$ be a non-null subset of $X$. Then the sub-soft set of $F_{E}$ over $Y$ denoted by $\left(F_{Y}, E\right)$, is defined as follows:

$$
F_{Y}(e)=Y \cap F(e) \forall e \in E .
$$

In other words $\left(F_{Y}, E\right)=\tilde{Y} \tilde{\cap} F_{E}$.

Definition 2.11. [29] Let $(X, \tau, E)$ be a soft topological space and $Y$ be a non-null subset of $X$. Then,

$$
\tau_{Y}=\left\{\left(F_{Y}, E\right): F_{E} \in \tau\right\}
$$

is said to be the soft relative topology on $Y$ and $\left(Y, \tau_{Y}, E\right)$ is called a soft subspace of $(X, \tau, E)$. 
Theorem 2.1. [29] Let $\left(Y, \tau_{Y}, E\right)$ be a soft subspace of a soft topological space $(X, \tau, E)$ and $F_{E} \in S S(X)_{E}$. Then,

(1) If $F_{E}$ is open soft set in $Y$ and $\tilde{Y} \in \tau$, then $F_{E} \in \tau$.

(2) $F_{E}$ is open soft set in $Y$ if and only if $F_{E}=\tilde{Y} \tilde{\cap} G_{E}$ for some $G_{E} \in \tau$.

(3) $F_{E}$ is closed soft set in $Y$ if and only if $F_{E}=\tilde{Y} \tilde{\cap} H_{E}$ for some $H_{E}$ is $\tau$-closed soft set.

Definition 2.12. $[6,10]$ Let $(X, \tau, E)$ be a soft topological space and $F_{E} \in S S(X)_{E}$. Then, $F_{E}$ is called a b-open soft set if $F_{E} \tilde{\subseteq} c l\left(\operatorname{int}\left(F_{E}\right)\right) \tilde{\cup} \operatorname{int}\left(\operatorname{cl}\left(F_{E}\right)\right)$. The set of all b-open soft sets is denoted by $B O S(X, \tau, E)$, or $B O S(X)$ and the set of all b-closed soft sets is denoted by $B C S(X, \tau, E)$, or $B C S(X)$.

Definition 2.13. $[6,10]$ Let $(X, \tau, E)$ be a soft topological space and $F_{E} \in S S(X)_{E}$. Then, the b-soft interior of $F_{E}$ is denoted by $\left.b \operatorname{Sint}\left(F_{E}\right)\right)$, where $\left.b \operatorname{Sint}\left(F_{E}\right)\right)=$ $\tilde{U}\left\{G_{E}: G_{E} \tilde{\subseteq} F_{E}, G_{E} \in B O S(X)\right\}$.

Also, the b-soft closure of $F_{E}$ is denoted by $\left.b \operatorname{Scl}\left(F_{E}\right)\right)$, where $\left.b \operatorname{Scl}\left(F_{E}\right)\right)=\tilde{\bigcap}\left\{H_{E}\right.$ : $\left.H_{E} \in B C S(X), F_{E} \tilde{\subseteq} H_{E}\right\}$.

Definition 2.14. [28] The soft b-boundary of a soft set $F_{E}$, denoted by b-Sbd( $\left.F_{E}\right)$, and is defined as b-Sbd( $\left.F_{E}\right)=b \operatorname{Scl}\left(F_{E}\right) \tilde{\cap} b \operatorname{Scl}\left(F_{E}^{c}\right)=b \operatorname{Scl}\left(F_{E}\right)-b \operatorname{Sint}\left(F_{E}\right)$.

Definition 2.15. [5] Let $S S(X)_{E}$ and $S S(Y)_{K}$ be two soft classes, $u: X \rightarrow Y$ and $p: E \rightarrow K$ be mappings. Then, the mapping $f_{p u}: S S(X)_{E} \rightarrow S S(Y)_{K}$ is defined as: for a soft set $(F, A)$ in $S S(X)_{E},\left(f_{p u}(F, A), B\right), B=p(A) \subseteq K$, is a soft set in $S S(Y)_{K}$ given by

$$
f_{p u}(F, A)(b)=u\left(\bigcup_{a \in p^{-1}(b) \cap A} F(a)\right)
$$

for $b \in B \subseteq K$. $\left(f_{p u}(F, A), B\right)$ is called a soft image of a soft set $(F, A)$. If $B=K$, then we shall write $\left(f_{p u}(F, A), K\right)$ as $f_{p u}(F, A)$. The soft function $f_{p u}$ is called surjective (resp. injective) if $p$ and $u$ are surjective (resp. injective).

Definition 2.16. [5] Let $f_{p u}: S S(X)_{E} \rightarrow S S(Y)_{K}$ be a mapping from a soft class $S S(X)_{E}$ to another soft class $S S(Y)_{K}$, and $(G, C)$ be a soft set in the soft class $S S(Y)_{K}$, where $C \subseteq K$. Let $u: X \rightarrow Y$ and $p: E \rightarrow K$ be mappings. Then, $\left(f_{p u}^{-1}(G, C), D\right), D=p^{-1}(C) \subseteq K$, is a soft set in the soft class $S S(X)_{E}$, defined as:

$$
f_{p u}^{-1}(G, C)(b)=u^{-1}(G(p(a)))
$$

for $a \in D \subseteq E$. $\left(f_{p u}^{-1}(G, C), D\right)$ is called a soft inverse image of $(G, C)$. Hereafter we shall write $\left(f_{p u}^{-1}(G, C), E\right)=f_{p u}^{-1}(G, C)$. 
Definition 2.17. $[10,30]$ Let $\left(X, \tau_{1}, E\right)$ and $\left(Y, \tau_{2}, K\right)$ be soft topological spaces and $f_{p u}: S S(X)_{E} \rightarrow S S(Y)_{K}$ be a function. Then, the function $f_{p u}$ is called

(1) Continuous soft if $f_{p u}^{-1}\left(G_{K}\right) \in \tau_{1} \forall G_{K} \in \tau_{2}$.

(2) Open soft if $f_{p u}\left(G_{E}\right) \in \tau_{2} \forall G_{E} \in \tau_{1}$.

(3) Closed soft if $f_{p u}\left(G_{E}\right) \in \tau_{2}^{c} \forall G_{E} \in \tau_{1}^{c}$.

(4) b-Continuous soft if $f_{p u}^{-1}\left(G_{K}\right) \in B O S(X) \forall G_{K} \in \tau_{2}$.

Definition 2.18. [23] A non-null soft subsets $F_{E}, G_{E}$ of a soft topological space $(X, \tau, E)$ are said to be soft separated sets if $c l\left(F_{E}\right) \tilde{\cap} G_{E}=F_{E} \tilde{\cap} c l\left(G_{E}\right)=\tilde{\phi}$. A soft topological space $(X, \tau, E)$ is said to be soft connected if and only if $\tilde{X}$ can not expressed as the soft union of two soft separated sets in $(X, \tau, E)$, which equivalent to, $\tilde{X}$ can not expressed as the soft union of two open soft sets in $(X, \tau, E)$. Otherwise, $(X, \tau, E)$ is said to be soft disconnected.

Definition 2.19. [16] A soft topological space $(X, \tau, E)$ is said to be soft hyperconnected if and only if every pair of non null proper open soft sets $F_{E}, G_{E}$, has a non null soft intersection, i.e $(X, \tau, E)$ is said to be soft hyperconnected iff $\forall F_{E}, G_{E} \in \tau$ we have $F_{E} \tilde{\cap} G_{E} \neq \tilde{\phi}$.

Definition 2.20. [8] Let $(X, \tau, E)$ be a soft topological space. A soft semi separation on $\tilde{X}$ is a pair of non-null proper semi open soft sets $F_{E}, G_{E}$ such that $F_{E} \tilde{\cap} G_{E}=\tilde{\phi}$ and $\tilde{X}=F_{E} \tilde{\cup} G_{E}$. A soft topological space $(X, \tau, E)$ is said to be soft semi connected if and only if there is no soft semi separations on $\tilde{X}$. Otherwise, $(X, \tau, E)$ is said to be soft semi disconnected.

Definition 2.21. [2] Let $(X, \tau, E)$ be a soft topological space. A soft $\beta$ - (resp. pre-) separation on $X$ is a pair of non-null proper $\beta$ - (resp. pre-) open soft sets $F_{E}, G_{E}$ such that $F_{E} \tilde{\cap} G_{E}=\tilde{\phi}$ and $\tilde{X}=F_{E} \tilde{\cup} G_{E}$. A soft topological space $(X, \tau, E)$ is said to be soft $\beta$ - (resp. pre-) connected if and only if there is no soft $\beta$ - (resp. pre-) separations on $\tilde{X}$. Otherwise, $(X, \tau, E)$ is said to be soft $\beta$ - (resp. pre-) disconnected.

\section{Soft b-separateness}

In this section, we will research the notion of soft b-separated sets in soft topological spaces and study its basic properties in detail.

Definition 3.1. Two non-null soft sets $G_{E}$ and $H_{E}$ of a soft topological space $(X, \tau, E)$ are said to be soft b-separated sets if $G_{E} \tilde{\cap} b S c l\left(H_{E}\right)=\tilde{\phi}$ and $b S c l\left(G_{E}\right) \tilde{\cap} H_{E}=$ $\tilde{\phi}$. Obviously, from the fact that $b c l\left(F_{E}\right) \tilde{\simeq} c l\left(F_{E}\right)$ for each soft set $F_{E} \in S S(X)_{E}$, every soft separated set is soft b-separated. But, the converse may not be true as shown in the following example. 
Example 3.1. Let $X=\left\{h_{1}, h_{2}, h_{3}, h_{4}\right\}, E=\left\{e_{1}, e_{2}\right\}, \tau=\left\{\tilde{X}, \tilde{\phi}, F_{1 E}, F_{2 E}, F_{3 E}\right\}$, where $F_{1 E}, F_{2 E}, F_{3 E}$ are soft sets over $X$ defined by:

$F_{1}\left(e_{1}\right)=\left\{h_{1}\right\}, \quad F_{1}\left(e_{2}\right)=\left\{h_{1}\right\}$,

$F_{2}\left(e_{1}\right)=\left\{h_{2}\right\}, \quad F_{2}\left(e_{2}\right)=\left\{h_{2}\right\}$,

$F_{3}\left(e_{1}\right)=\left\{h_{1}, h_{2}\right\}, \quad F_{3}\left(e_{2}\right)=\left\{h_{1}, h_{2}\right\}$.

The soft sets $H_{E}$ and $G_{E}$, which defined by:

$H\left(e_{1}\right)=\left\{h_{1}\right\}, \quad H\left(e_{2}\right)=\left\{h_{1}\right\}$,

$G\left(e_{1}\right)=\left\{h_{3}, h_{4}\right\}, \quad G\left(e_{2}\right)=\left\{h_{3}, h_{4}\right\}$, are soft b-separated but not soft separated.

Definition 3.2. A soft set $F_{E}$ of a soft topological space $(X, \tau, E)$ is said to be b-clopen soft set if it is both b-open soft set and b-closed soft set.

Proposition 3.1. (1) Each two soft b-separated sets are always disjoint

(2) Each two disjoint soft sets, in which both of them either b-open soft sets or $b$-closed soft sets, are soft b-separated.

Proof. Clear from Definition 3.1.

Theorem 3.1. Let $G_{E}$ and $H_{E}$ be non-null soft sets of a soft topological space $(X, \tau, E)$. Then, the following statements hold:

(1) If $G_{E}$ and $H_{E}$ are soft b-separated, $G_{1 E} \tilde{\subseteq} G_{E}$ and $H_{1 E} \tilde{\subseteq} H_{E}$, then $G_{1 E}$ and $H_{1 E}$ are soft b-separated sets.

(2) If $G_{E}$ and $H_{E}$ are b-open soft sets, $U_{E}=G_{E} \tilde{\cap}\left(\tilde{X}-H_{E}\right)$ and $V_{E}=H_{E} \tilde{\cap}(\tilde{X}-$ $\left.G_{E}\right)$, then $U_{E}$ and $V_{E}$ are soft b-separated sets.

Proof.

(1) Since $G_{1 E} \tilde{\subseteq} G_{E}$. Then, $b S c l\left(G_{1 E}\right) \tilde{\subseteq} b S c l\left(G_{E}\right)$. Hence,

$$
H_{1 E} \tilde{\cap} b \operatorname{Scl}\left(G_{1 E}\right) \tilde{\tilde{\subseteq}} H_{E} \tilde{\cap} b \operatorname{Scl}\left(G_{E}\right)=\tilde{\phi} .
$$

Similarly, $G_{1 E} \tilde{\cap} b S c l\left(H_{1 E}\right)=\tilde{\phi}$. Thus, $G_{1 E}$ and $H_{1 E}$ are soft b-separated sets.

(2) Let $G_{E}$ and $H_{E}$ be b-open soft sets. Then, $\left(\tilde{X}-G_{E}\right)$ and $\left(\tilde{X}-H_{E}\right)$ are b-closed soft sets. Assume that, $U_{E}=G_{E} \tilde{\cap}\left(\tilde{X}-H_{E}\right)$ and $V_{E}=H_{E} \tilde{\cap}\left(\tilde{X}-G_{E}\right)$. Then, $U_{E} \tilde{\subseteq}\left(\tilde{X}-H_{E}\right)$ and $V_{E} \tilde{\subseteq}\left(\tilde{X}-G_{E}\right)$. Hence, $b S c l\left(U_{E}\right) \tilde{\subseteq}\left(\tilde{X}-H_{E}\right) \tilde{\subseteq}\left(\tilde{X}-V_{E}\right)$ and $b S c l\left(V_{E}\right) \tilde{\subseteq} \tilde{X}-G_{E} \tilde{\subseteq}\left(\tilde{X}-U_{E}\right)$. Consequently, $b S c l\left(U_{E}\right) \tilde{\cap} V_{E}=\tilde{\phi}$ and $\operatorname{bScl}\left(V_{E}\right) \tilde{\cap} U_{E}=\tilde{\phi}$. Therefore, $U_{E}$ and $V_{E}$ are soft b-separated sets.

Theorem 3.2. Any two soft sets $G_{E}$ and $H_{E}$ of a soft topological space $(X, \tau, E)$ are soft b-separated sets if and only if there exist b-open soft sets $U_{E}$ and $V_{E}$ such that $G_{E} \tilde{\subseteq} U_{E}, H_{E} \tilde{\subseteq} V_{E}$ and $G_{E} \tilde{\cap} V_{E}=\tilde{\phi}, H_{E} \tilde{\cap} U_{E}=\tilde{\phi}$.

Proof. Let $G_{E}$ and $H_{E}$ be soft b-separated sets. Then, $G_{E} \tilde{\cap} b S c l\left(H_{E}\right)=\tilde{\phi}$ and $b S c l\left(G_{E}\right) \tilde{\cap} H_{E}=\tilde{\phi}$. Let $V_{E}=\tilde{X}-b \operatorname{Scl}\left(G_{E}\right)$ and $U_{E}=\tilde{X}-b S c l\left(H_{E}\right)$. Thus, $U_{E}$ and $V_{E}$ are b-open soft sets such that $G_{E} \tilde{\subseteq} U_{E}, H_{E} \tilde{\subseteq} V_{E}, G_{E} \tilde{\cap} V_{E}=\tilde{\phi}$ and $H_{E} \tilde{\cap} U_{E}=\tilde{\phi}$. 
On the other hand, let $U_{E}, V_{E}$ be b-open soft sets such that $G_{E} \tilde{\subseteq} U_{E}, H_{E} \tilde{\subseteq} V_{E}$ and $G_{E} \tilde{\cap} V_{E}=\tilde{\phi}, H_{E} \tilde{\cap} U_{E}=\tilde{\phi}$. Since $\left(\tilde{X}-V_{E}\right)$ and $\left(\tilde{X}-U_{E}\right)$ are b-closed soft sets. Then, $\left.b S c l\left(G_{E}\right) \tilde{\subseteq} \tilde{(} X_{E}-V_{E}\right) \tilde{\subseteq} \tilde{(}\left(X_{E}-H_{E}\right)$ and $b S c l\left(H_{E}\right) \tilde{\subseteq} \tilde{(}\left(X_{E}-U_{E}\right) \tilde{\subseteq} \tilde{(}\left(X_{E}-G_{E}\right)$. Thus, $b \operatorname{Scl}\left(G_{E}\right) \tilde{\cap} H_{E}=\tilde{\phi}$ and $b S c l\left(H_{E}\right) \tilde{\cap} G_{E}=\tilde{\phi}$. This means that, $G_{E}$ and $H_{E}$ are soft b-separated sets.

\section{Soft b-connectedness}

In this section, we will research the notion of soft b-connectedness in soft topological spaces be means of b-open soft sets, b-closed soft sets, soft -separated sets and study its basic properties.

Definition 4.1. Let $(X, \tau, E)$ be a soft topological space. A soft b-separation of $\tilde{X}$ is a pair of non-null proper b-open soft sets in $\tau$ such that $F_{E} \tilde{\cap} G_{E}=\tilde{\phi}$ and $\tilde{X}=F_{E} \tilde{\cup} G_{E}$.

Definition 4.2. A soft topological space $(X, \tau, E)$ is said to be b-soft connected if and only if there is no soft b-separations of $\tilde{X}$. If $(X, \tau, E)$ has such soft bseparations, then $(X, \tau, E)$ is said to be soft b-disconnected.

Theorem 4.1. Let $(X, \tau, E)$ be a soft topological space, then the following statements are equivalent:

(1) $\tilde{X}$ is soft b-connected.

(2) $\tilde{X}$ cannot be expressed as a soft union of two non-null disjoint b-open soft sets.

(3) $\tilde{X}$ cannot be expressed as a soft union of two non-null disjoint b-closed soft sets.

(4) There is no proper b-clopen soft set in $(X, \tau, E)$.

(5) $\tilde{X}$ cannot be expressed as a soft union of two non-null b-separated soft sets.

Proof.

$(1) \Leftrightarrow(2)$ It is obvious from Definition 4.2.

$(2) \Rightarrow(3)$ Suppose that $\tilde{X}=F_{E} \tilde{\cup} G_{E}$ for some b-closed soft sets $F_{E}$ and $G_{E}$ such that $F_{E} \tilde{\cap} G_{E}=\tilde{\phi}$. Then, $F_{E}=\left(G_{E}\right)^{c}$ which is b-open soft set, $\tilde{X}=G_{E} \tilde{\cup} G_{E}^{c}$ and $G_{E} \tilde{\cap} G_{E}^{c}=\tilde{\phi}$, which is a contradiction with (2).

(3) $\Rightarrow$ (4) Suppose that there is a proper b-clopen soft subset $F_{E}$ of $\tilde{X}$. Then, $F_{E}^{c}$ is b-clopen soft set, where $\tilde{X}=F_{E} \tilde{\cup} F_{E}^{c}$ and $F_{E} \tilde{\cap} F_{E}^{c}=\tilde{\phi}$, which is a contradiction with (3). 
(4) $\Rightarrow$ (3) Suppose that $\tilde{X}=F_{E} \tilde{\cup} G_{E}$ for some b-closed soft sets $F_{E}$ and $G_{E}$ such that $F_{E} \tilde{\cap} G_{E}=\tilde{\phi}$. Then, $F_{E}=G_{E}^{c}$ and $G_{E}=F_{E}^{c}$. Thus, $F_{E}$ and $G_{E}$ are proper b-clopen soft sets, which is a contradiction with (4).

(3) $\Rightarrow(5)$ Suppose that $\tilde{X}=H_{E} \tilde{\cup} G_{E}$ for some b-separated soft sets $H_{E}$ and $G_{E}$. Then, $G_{E} \tilde{\cap} b S c l\left(H_{E}\right)=\tilde{\phi}$ and $b \operatorname{Scl}\left(G_{E}\right) \tilde{\cap} H_{E}=\tilde{\phi}$. It follows that, $H_{E} \tilde{\cap} G_{E}=$ $\tilde{\phi}$. Hence, $H_{E}=G_{E}^{c}$ and $G_{E}=H_{E}^{c}$. Therefore, $\operatorname{bScl}\left(H_{E}\right) \tilde{\subseteq} G_{E}^{c}=H_{E}$ and $b \operatorname{Scl}\left(G_{E}\right) \tilde{\subseteq} H_{E}^{c}=G_{E}$. But, $H_{E} \tilde{\subseteq} b S c l\left(H_{E}\right)$ and $G_{E} \tilde{\subseteq} b S c l\left(G_{E}\right)$. Thus, $H_{E}$ and $G_{E}$ are b-closed soft sets, which is a contradiction with (3).

$(5) \Rightarrow(1)$ Suppose that $\tilde{X}=F_{E} \tilde{\cup} G_{E}$ for some b-open soft sets $F_{E}$ and $G_{E}$ such that $F_{E} \tilde{\cap} G_{E}=\tilde{\phi}$. Then, $F_{E}=G_{E}^{c}$ and $G_{E}=F_{E}^{c}$. Thus, $F_{E}$ and $G_{E}$ are b-clopen soft sets. Hence, $F_{E}$ and $G_{E}$ are b-separated soft sets, which is a contradiction with (5).

Corollary 4.1. Let $(X, \tau, E)$ be a soft topological space, then the following statements are equivalent:

(1) $\tilde{X}$ is soft b-connected.

(2) If $\tilde{X}=F_{E} \tilde{\cup} G_{E}$ for some b-open soft sets $F_{E}$ and $G_{E}$ such that $F_{E} \tilde{\cap} G_{E}=\tilde{\phi}$. Then, either $F_{E}=\tilde{\phi}$ or $G_{E}=\tilde{\phi}$.

(3) If $\tilde{X}=F_{E} \tilde{\cup} G_{E}$ for some b-closed soft sets $F_{E}$ and $G_{E}$ such that $F_{E} \tilde{\cap} G_{E}=\tilde{\phi}$. Then, either $F_{E}=\tilde{\phi}$ or $G_{E}=\tilde{\phi}$.

Proof. Obvious from Theorem 4.1.

Definition 4.3. A soft subset $F_{E}$ of a soft topological space $(X, \tau, E)$ is soft bconnected, if it is soft b-connected as a soft subspace. In other words, a soft subset $F_{E}$ of a soft topological space $(X, \tau, E)$ is said to be soft b-connected relative to $\tilde{X}$ if there is not exist two soft b-separated subsets $H_{E}$ and $G_{E}$ relative to $\tilde{X}$ and $F_{E}=H_{E} \tilde{\cup} G_{E}$. Otherwise, $F_{E}$ is said to be soft b-disconnected.

Remark 4.1. Each soft disconnected set is soft b-disconnected, but not conversely as shown in the following examples.

Example 4.1. (1) In Example 3.1, the soft set $G_{E}$, where $G\left(e_{1}\right)=\left\{h_{1}, h_{3}, h_{4}\right\}, \quad G\left(e_{2}\right)=\left\{h_{1}, h_{3}, h_{4}\right\}$, is soft connected set, but not soft b-connected.

(2) Any soft space with the indiscrete soft topology is soft connected, but not soft bconnected, because the family of b-open soft sets establish a discrete soft topology.

Corollary 4.2. Let $\left(X, \tau_{1}, E\right)$ and $\left(X, \tau_{2}, E\right)$ be soft topological spaces and $\tau_{2} \subseteq \tau_{1}$. If $\left(X, \tau_{1}, E\right)$ is soft b-connected, then $\left(X, \tau_{2}, E\right)$ is soft b-connected.

Proof. It is Obvious. 
Definition 4.4. Let $(X, \tau, E)$ be a soft topological space and $Z_{E} \tilde{\subseteq} \tilde{X}$ with $x \in$ $Z_{E}$. Then, the soft b-component of $Z_{E}$ w.r.t. $x_{\alpha}$ is the maximal of all soft bconnected subspaces of $\left(Z, \tau_{Z}, E\right)$ containing $x_{\alpha}$ and denoted by $\tilde{S C_{b}}\left[Z_{E}, x_{\alpha}\right]$ or $\tilde{S C_{b}}\left(Z_{E}, x_{\alpha}\right)$ for short, i.e

$$
\tilde{S C_{b}}\left(Z_{E}, x_{\alpha}\right)=\tilde{\cup}\left\{Y_{E} \tilde{\subseteq} Z_{E}: x_{\alpha} \in Y_{E}, Y_{E} \text { is soft b-connected }\right\} \text {. }
$$

Theorem 4.2. Every soft b-component of a soft topological space $(X, \tau, E)$ is a maximal soft b-connected subset of $\tilde{X}$.

Proof. It is obvious from Definition 4.4.

Corollary 4.3. The soft topological space $(X, \tau, E)$ is soft b-connected if and only if it is a soft b-component on $\tilde{X}$.

Proof. It is clear.

Theorem 4.3. If the non-null soft sets $G_{E}$ and $H_{E}$ of a soft topological space $(X, \tau, E)$ are soft b-separated, then $\left(G_{E} \tilde{\cup} H_{E}\right)$ is soft b-disconnected.

Proof. Let $G_{E}$ and $H_{E}$ be non-null soft b-separated sets, then there exist b-open soft sets $U_{E}$ and $V_{E}$ such that $G_{E} \tilde{\widetilde{C}} U_{E}, H_{E} \tilde{\subseteq} V_{E}$ and $G_{E} \tilde{\cap} V_{E}=\tilde{\phi}$, $H_{E} \tilde{\cap} U_{E}=\tilde{\phi}$ follows immediately from Theorem 3.2. Hence, $\left(G_{E} \tilde{\cup} H_{E}\right) \tilde{\cap} U_{E}=G_{E}$ and $\left(G_{E} \tilde{\cup} H_{E}\right) \tilde{\cap} V_{E}=H_{E}$. Consequently, $G_{E} \tilde{\cup} H_{E}$ is soft b-disconnected.

Next, we characterized soft b-connectedness in terms of soft boundaries as follows:

Theorem 4.4. A soft topological space $(X, \tau, E)$ is soft b-connected if and only if every non-null proper subset has a non-null soft b-boundary.

Proof. Let $(X, \tau, E)$ be soft b-disconnected, then $(X, \tau, E)$ has a proper bclopen soft set $F_{E}$. Then, $b \operatorname{Scl}\left(F_{E}\right)=F_{E}=b \operatorname{Sint}\left(F_{E}\right)=\tilde{X}-b \operatorname{Scl}\left(\tilde{X}-F_{E}\right)$. Therefore, b-Sbd $\left(F_{E}\right)=b \operatorname{Scl}\left(F_{E}\right) \tilde{n} b \operatorname{Scl}\left(\tilde{X}-F_{E}\right)=\tilde{\phi}$. Therefore, $F_{E}$ has an empty soft b-boundary.

Conversely, suppose that a non-null proper soft subset $F_{E}$ has an empty soft bboundary. Then, b-Sbd( $\left.F_{E}\right)=\operatorname{bScl}\left(F_{E}\right) \tilde{n} b \operatorname{Scl}\left(\tilde{X}-F_{E}\right)=\tilde{\phi}$. Consequently, $b \operatorname{Scl}\left(F_{E}\right) \tilde{\subseteq}\left[\tilde{X}-b \operatorname{Scl}\left(\tilde{X}-F_{E}\right)\right]=b \operatorname{Sint}\left(F_{E}\right)$, and thus $F_{E} \tilde{\subseteq} b \operatorname{Scl}\left(F_{E}\right) \tilde{\subseteq} b \operatorname{Sint}\left(F_{E}\right) \tilde{\subseteq} F_{E}$. Thus, $F_{E}$ is a proper b-clopen soft set and consequently, $(X, \tau, E)$ is soft b-disconnected.

Theorem 4.5. Let $\left(Z, \tau_{Z}, E\right)$ be a soft subspace of a soft topological space $(X, \tau, E)$ and $F_{1 E}, F_{2 E} \tilde{\subseteq} Z_{E} \tilde{\subseteq} \tilde{X}$. Then, $F_{1 E}, F_{2 E}$ are soft b-separated on $\tau_{Z}$ if and only if $F_{1 E}, F_{2 E}$ are soft b-separated on $\tau$.

Proof. Suppose that $F_{1 E}, F_{2 E}$ are soft b-separated on $\tau_{Z} \Leftrightarrow b S c l_{\tau_{Z}} F_{1 E} \tilde{\cap} F_{2 E}=$ $\tilde{\phi}$ and $F_{1 E} \tilde{\cap} b S c l_{\tau_{Z}} F_{2 E}=\tilde{\phi} \Leftrightarrow\left[b S c l_{\tau} F_{1 E} \tilde{\cap} Z_{E}\right] \tilde{\cap} F_{2 E}=b S c l_{\tau} F_{1 E} \tilde{\cap} F_{2 E}=\tilde{\phi}$ and $\left[b S c l_{\tau} F_{2 E} \tilde{\cap} Z_{E}\right] \tilde{\cap} F_{1 E}=b S c l_{\tau} F_{2 E} \tilde{\cap} F_{1 E}=\tilde{\phi} \Leftrightarrow F_{1 E}, F_{2 E}$ are soft b-separated on $\tau$. 
Theorem 4.6. Let $Z_{E}$ be a soft subset of a soft topological space $(X, \tau, E)$. Then, $Z_{E}$ is soft b-connected relative to $(X, \tau, E)$ if and only if it is soft $b$-connected relative to $\left(Z, \tau_{Z}, E\right)$.

Proof. Suppose that $Z_{E}$ is soft b-disconnected relative to $\left(Z, \tau_{Z}, E\right) \Leftrightarrow Z_{E}=$ $F_{1 E} \tilde{\cup} F_{2 E}$, where $F_{1 E}$ and $F_{2 E}$ are soft b-separated on $\tau_{Z} \Leftrightarrow Z_{E}=F_{1 E} \tilde{\cup} F_{2 E}$, where $F_{1 E}$ and $F_{2 E}$ are soft b-separated on $\tau_{Z}$ from Theorem $4.5 \Leftrightarrow Z_{E}$ is soft b-disconnected relative to $(X, \tau, E)$.

Theorem 4.7. Let $\left(Z, \tau_{Z}, E\right)$ be a soft b-connected subspace of a soft topological space $(X, \tau, E)$ and $F_{E}, G_{E}$ are soft b-separated sets of $\tilde{X}$ with $Z_{E} \tilde{\subseteq} F_{E} \tilde{\cup} G_{E}$, then either $Z_{E} \tilde{\subseteq} F_{E}$ or $Z_{E} \tilde{\subseteq} G_{E}$.

Proof. Let $Z_{E} \tilde{\subseteq} F_{E} \tilde{\cup} G_{E}$ for some soft b-separated sets $F_{E}, G_{E}$ on $\tau$. Since $Z_{E}=\left(Z_{E} \tilde{\cap} F_{E}\right) \tilde{\cup}\left(Z_{E} \tilde{\cap} G_{E}\right)$. Then, $\left(Z_{E} \tilde{\cap} F_{E}\right) \tilde{\cup} b S c l_{\tau}\left(Z_{E} \tilde{\cap} G_{E}\right) \tilde{\subseteq} F_{E} \tilde{\cap} b S c l_{\tau}\left(G_{E}\right)=$ $\tilde{\phi}$. Also, $b S c l_{\tau}\left(Z_{E} \tilde{\cap} F_{E}\right) \tilde{\cup}\left(Z_{E} \tilde{\cap} G_{E}\right) \tilde{\tilde{\subseteq}} c l_{\tau}\left(F_{E}\right) \tilde{\cap} G_{E}=\tilde{\phi}$. If $Z_{E} \tilde{\cap} F_{E}$ and $Z_{E} \tilde{\cap} G_{E}$ are non-null soft sets. Then, $Z_{E}$ is soft b-disconnected, which is a contradiction with the hypothesis. Thus, either $Z_{E} \tilde{\cap} F_{E}=\tilde{\phi}$ or $Z_{E} \tilde{\cap} G_{E}=\tilde{\phi}$. It follows that, $Z_{E}=Z_{E} \tilde{\cap} F_{E}$ or $Z_{E}=Z_{E} \tilde{\cap} G_{E}$. Therefore, $Z_{E} \tilde{\subseteq} F_{E}$ or $Z_{E} \tilde{\subseteq} G_{E}$.

Theorem 4.8. The soft b-closure of a soft b-connected set is soft b-connected.

Proof. Assume that, $b \operatorname{Scl}\left(F_{E}\right)$ is soft b-disconnected. Then, there are two nonnull soft b-separated sets $G_{E}$ and $H_{E}$ such that $b \operatorname{Scl}\left(F_{E}\right)=G_{E} \tilde{\cup} H_{E}$. Since $F_{E}$ is soft b-connected. By using Theorem 4.7, either $F_{E} \tilde{\subseteq} G_{E}$ or $F_{E} \tilde{\subseteq} H_{E}$. If $F_{E} \tilde{\subseteq} G_{E}$, then $b \operatorname{Scl}\left(F_{E} \tilde{\subseteq} b S c l\left(G_{E}\right)\right.$ and so $b \operatorname{Scl}\left(F_{E} \tilde{\cap} H_{E}=\tilde{\phi}\right.$. But, $H_{E} \tilde{\subseteq} b S c l\left(F_{E}\right)$. Therefore, $H_{E}=\tilde{\phi}$ which is a contradiction. Similarly, if $F_{E} \tilde{\subseteq} H_{E}$, we can get $G_{E}=\tilde{\phi}$, which is also a contradiction. Consequently, $b \operatorname{Scl}\left(F_{E}\right)$ is soft b-connected.

Theorem 4.9. Every soft b-component of a soft topological space $(X, \tau, E)$ is $b$ closed soft set.

Proof. It is obvious from Definition 4.4 and from the fact that the soft b-closure of a soft b-connected set is a soft b-connected.

Theorem 4.10. Let $(X, \tau, E)$ be a soft topological space. Then:

(1) Each soft point $x_{\alpha} \tilde{\in} \tilde{X}$ is contained in exactly one soft b-component of $\tilde{X}$.

(2) Any two soft b-components w.r.t. two different soft points are either disjoint or identical.

Proof.

(1) Let $x_{\alpha} \tilde{\in} \tilde{X}$ and consider the collection $\tilde{S C_{b}}=\left\{Z_{E} \tilde{\subseteq} \tilde{X}: x_{\alpha} \tilde{\in} Z_{E}, Z_{E}\right.$ is soft b-connected $\}$. Then, we have:

(a) $\tilde{S C_{b}} \neq \tilde{\phi}$, for the soft point $x_{\alpha}$ is a soft b-connected subset of $\tilde{X}$ containing $x_{\alpha}$. Then, $x_{\alpha} \in \tilde{S C_{b}}$. 
(b) $\tilde{\cap}\left\{Z_{E} \tilde{\subseteq} \tilde{X}: x_{\alpha} \tilde{\in} Z_{E}: Z_{E}\right.$ is soft b-connected $\} \neq \tilde{\phi}$. Since $x_{\alpha} \tilde{\in} Z_{E} \forall Z_{E} \in$ $S C_{b}$.

(c) The soft set $\tilde{\cup}\left\{Z_{E} \tilde{\subseteq} \tilde{X}: x_{\alpha} \tilde{\in} Z_{E}, Z_{E}\right.$ is soft b-connected $\}$, having a nonnull soft intersection, is soft b-connected subset of $\tilde{X}$ containing $x_{\alpha}$.

(d) $\tilde{\cup}\left\{Z_{E} \tilde{\subseteq} \tilde{X}: x_{\alpha} \tilde{\in} Z_{E}, Z_{E}\right.$ is soft b-connected $\}$ is the largest soft b-connected subset of $\tilde{X}$ containing $x_{\alpha}$, which is the soft b-component $\tilde{S} C_{b}\left(\tilde{X}, x_{\alpha}\right)$ of $\tilde{X}$ w.r.t $x_{\alpha}$ and containing $x_{\alpha}$ from Definition 4.4.

Now, suppose $\tilde{S C}_{b}^{*}\left(\tilde{X}, x_{\alpha}\right)$ be another soft b-component containing $x_{\alpha}$, then $\tilde{S C_{b}^{*}}\left(\tilde{X}, x_{\alpha}\right)$ is a soft b-connected subset of $\tilde{X}$ containing $x_{\alpha}$, but $\tilde{S C_{b}}\left(\tilde{X}, x_{\alpha}\right)$ is a soft b-component, then $\tilde{S C_{b}}\left(\tilde{X}, x_{\alpha}\right)$ is the largest soft b-connected subset of $\tilde{X}$ containing $x_{\alpha}$, consequently, $\tilde{S C_{b}^{*}}\left(\tilde{X}, x_{\alpha}\right) \tilde{\subseteq} \tilde{S C_{b}}\left(\tilde{X}, x_{\alpha}\right)$. Similarly, $\tilde{S C_{b}}\left(\tilde{X}, x_{\alpha}\right) \tilde{\subseteq} \tilde{S C}_{b}^{*}\left(\tilde{X}, x_{\alpha}\right)$, and hence $x_{\alpha}$ is contained in exactly one soft bcomponent of $\tilde{X}$.

(2) Let $\tilde{S C_{b}}\left(\tilde{X}, x_{1 \alpha}\right), \tilde{S C_{b}}\left(\tilde{X}, x_{2 \alpha}\right)$ be the soft b-components of $x_{\alpha}$ w.r.t two different soft points $x_{1 \alpha}, x_{2 \alpha}$ of $\tilde{X}$ with $x_{1 \alpha} \neq x_{2 \alpha}$ respectively.

If $\tilde{S C_{b}}\left(\tilde{X}, x_{1 \alpha}\right) \tilde{\cap} \tilde{S C} C_{b}\left(\tilde{X}, x_{2 \alpha}\right)=\tilde{\phi}$, then we get the proof.

So, let $\tilde{S C_{b}}\left(\tilde{X}, x_{1 \alpha}\right) \tilde{\cap} \tilde{S C_{b}}\left(\tilde{X}, x_{2 \alpha}\right) \neq \phi$. We may choose a

$x_{\beta} \in \tilde{S C_{b}}\left(\tilde{X}, x_{1 \alpha}\right) \tilde{\cap} \tilde{S C_{b}}\left(\tilde{X}, x_{2 \alpha}\right)$. Clearly, $x_{\beta} \in \tilde{S C_{b}}\left(\tilde{X}, x_{1 \alpha}\right)$ and $x_{\beta} \in$ $\tilde{S C_{b}}\left(\tilde{X}, x_{2 \alpha}\right)$, which mean that $\tilde{S C_{b}}\left(\tilde{X}, x_{1 \alpha}\right)$ is the largest soft b-connected subset of $\tilde{X}$ containing $x_{\beta}, \tilde{S C_{b}}\left(\tilde{X}, x_{2 \alpha}\right)$ is the largest soft b-connected subset of $\tilde{X}$ containing $x_{\beta}$. Therefore, $\tilde{S C_{b}}\left(\tilde{X}, x_{1 \alpha}\right)=\tilde{S C_{b}}\left(\tilde{X}, x_{2 \alpha}\right)$, and hence $\tilde{S C_{b}}\left(\tilde{X}, x_{1 \alpha}\right)$ and $\tilde{S C_{b}}\left(\tilde{X}, x_{2 \alpha}\right)$ are identical. This completes the proof.

Theorem 4.11. Let $F_{E}$ be soft b-connected subsets of a soft topological space $(X, \tau, E)$ and $G_{E}$ be a soft set such that $F_{E} \tilde{\subseteq} G_{E} \tilde{\subseteq} b S c l\left(F_{E}\right)$, then $G_{E}$ is soft $b$ connected.

Proof. Let $G_{E}$ be a soft b-disconnected, then there exist two non-null b-open soft sets $U_{E}$ and $V_{E}$ such that $G_{E}=U_{E} \tilde{\cup} V_{E}$. Since $F_{E} \tilde{\subseteq} G_{E}$ and $F_{E}$ be soft b-connected, then by using Lemma 4.10 either $F_{E} \tilde{\widetilde{\subseteq}} U_{E}$ or $F_{E} \tilde{\subseteq} V_{E}$. If $F_{E} \tilde{\subseteq} U_{E}$, then $b \operatorname{Scl}\left(F_{E}\right) \tilde{\subseteq} b S c l\left(U_{E}\right)$ and so $b \operatorname{Scl}\left(F_{E}\right) \tilde{\cap} V_{E}=\tilde{\phi}$ i.e, $V_{E} \tilde{\subseteq} \tilde{X}-b \operatorname{Scl}\left(F_{E}\right)$, but $V_{E} \tilde{\subseteq} G_{E} \tilde{\subseteq} b \operatorname{Scl}\left(F_{E}\right)$. Thus, $V_{E}=\tilde{\phi}$, which is a contradiction, and so $G_{E}$ is soft bconnected. Similarly, if $F_{E} \tilde{\subseteq} V_{E}$, thus $U_{E}=\tilde{\phi}$ this is a contradiction. Consequently, $G_{E}$ is soft b-connected.

Theorem 4.12. Let $\left(Z, \tau_{Z}, E\right)$ be a soft b-connected subspace of a soft b-connected topological space $(X, \tau, E)$ such that $Z_{E}^{c}$ is the soft union of two soft b-separated sets $F_{E}, G_{E}$ of $\tilde{X}$, then $Z_{E} \tilde{\cup} F_{E}$ and $Z_{E} \tilde{\cup} G_{E}$ are soft b-connected.

Proof. Suppose that $Z_{E} \tilde{\cup} F_{E}$ is soft b-disconnected relative to $(X, \tau, E)$. Then, there exist two non-null soft b-separated sets $K_{E}$ and $H_{E}$ of $\tilde{X}$ such that $Z_{E} \tilde{\cup} F_{E}=$ $K_{E} \tilde{\cup} H_{E}$. Since $Z_{E}$ is soft b-connected and $Z_{E} \tilde{\subseteq} Z_{E} \tilde{\cup} F_{E}=K_{E} \tilde{\cup} H_{E}$. It follows that, either $Z_{E} \tilde{\subseteq} K_{E}$ or $Z_{E} \tilde{\subseteq} H_{E}$ from Theorem 4.7. Suppose $Z_{E} \tilde{\subseteq} K_{E}$. Since $Z_{E} \tilde{\cup} F_{E}=$ $K_{E} \tilde{\cup} H_{E}$ and $Z_{E} \tilde{\subseteq} K_{E}$, implies that $Z_{E} \cup F_{E} \tilde{\subseteq} K_{E} \cup F_{E}$. So, $K_{E} \tilde{\cup} H_{E} \tilde{\subseteq} K_{E} \tilde{\cup} F_{E}$. Hence, 
$H_{E} \tilde{\subseteq} F_{E}, G_{E} \tilde{\simeq} K_{E}$. By Theorem 3.1 (1), $H_{E}, G_{E}$ are soft b-separated. Now,

$$
b S c l_{\tau} H_{E} \tilde{\cap}\left[K_{E} \tilde{\cup} G_{E}\right]=\left[b S c l_{\tau} H_{E} \tilde{\cap} K_{E}\right] \tilde{\cup}\left[b S c l_{\tau} H_{E} \tilde{\cap} G_{E}\right]=\tilde{\phi}
$$

and $H_{E} \tilde{\cap} c l_{\tau}\left[K_{E} \tilde{\cup} G_{E}\right]=H_{E} \tilde{\cap}\left[c l_{\tau} K_{E} \tilde{\cup} c l_{\tau} G_{E}\right]=\left[H_{E} \tilde{\cap} c l_{\tau} K_{E}\right] \tilde{\cup}\left[H_{E} \tilde{\cap} c l_{\tau} G_{E}\right]=\tilde{\phi}$. It follows that, $H_{E}$ and $\left[K_{E} \tilde{\cup} G_{E}\right]$ are soft b-separated on $\tau$. Since $Z_{E}^{c}=F_{E} \tilde{\cup} G_{E}$. Then,

$\tilde{X}=Z_{E} \tilde{\cup} Z_{E}^{c}=Z_{E} \tilde{\cup}\left[F_{E} \tilde{\cup} G_{E}\right]=\left[Z_{E} \tilde{\cup} F_{E}\right] \tilde{\cup} G_{E}=\left[K_{E} \tilde{\cup} H_{E}\right] \tilde{\cup} G_{E}=H_{E} \tilde{\cup}\left[K_{E} \tilde{\cup} G_{E}\right]$.

Thus, $\tilde{X}$ is the soft union of two non null soft b-separated sets $H_{E}$ and $K_{E} \tilde{\cup} G_{E}$, which is a contradiction. A similar contradiction will arise if $Z_{E} \tilde{\simeq} H_{E}$. Hence, $Z_{E} \tilde{\cup} F_{E}$ is soft b-connected. Similarly, we can prove that $Z_{E} \tilde{\cup} G_{E}$ is soft b-connected.

Theorem 4.13. If $Z_{E}, Y_{E}$ are soft b-connected sets such that none of them is soft b-separated sets, then $Z_{E} \tilde{\cup} Y_{E}$ is soft b-connected set.

Proof. Let $\left(Z, \tau_{Z}, E\right)$ and $\left(Y, \tau_{Y}, E\right)$ be soft b-connected subspaces of $\tilde{X}$ such that $Z_{E} \tilde{\cup} Y_{E}$ is not soft b-connected relative to $(X, \tau, E)$. Then, there exist two non-null soft b-separated sets $K_{E}$ and $H_{E}$ of $\tilde{X}$ such that $Z_{E} \tilde{\cup} Y_{E}=K_{E} \tilde{\cup} H_{E}$. Since $Z_{E}, Y_{E}$ are soft b-connected and $Z_{E}, Y_{E} \tilde{\subseteq} Z_{E} \tilde{\cup} Y_{E}=K_{E} \tilde{\cup} H_{E}$. Then, either $Z_{E} \tilde{\subseteq} K_{E}$ or $Z_{E} \tilde{\subseteq} H_{E}$, also, either $Y_{E} \tilde{\simeq} K_{E}$ or $Y_{E} \tilde{\subseteq} H_{E}$ from Theorem 4.7. If $Z_{E} \tilde{\subseteq} K_{E}$ or $Z_{E} \tilde{\subseteq} H_{E}$. Then, $Z_{E} \tilde{\cap} H_{E} \tilde{\subseteq} K_{E} \tilde{\cap} H_{E}=\tilde{\phi}$ or $Z_{E} \tilde{\cap} K_{E} \tilde{\subseteq} H_{E} \tilde{\cap} K_{E}=\tilde{\phi}$. Therefore, $\left[Z_{E} \tilde{\cup} Y_{E}\right] \tilde{\cap} K_{E}=\left[Z_{E} \tilde{\cap} K_{E}\right] \tilde{\cup}\left[Y_{E} \tilde{\cap} K_{E}\right]=\tilde{\phi} \tilde{\cup}\left[Y_{E} \tilde{\cap} K_{E}\right]=Y_{E} \tilde{\cap} K_{E}=Y_{E}$, since $Y_{E} \tilde{\subseteq} K_{E}$. Similarly, if $Y_{E} \tilde{\subseteq} K_{E}$ or $Y_{E} \tilde{\subseteq} H_{E}$. We can get, $\left[Z_{E} \tilde{\cup} Y_{E}\right] \tilde{\cap} H_{E}=Z_{E}$. Now,

$$
\begin{gathered}
\tilde{\cap} b S c l_{\tau}\left[\left(Z_{E} \tilde{\cup} Y_{E}\right) \tilde{\cap} K_{E}\right] \tilde{\subseteq}\left[\left(Z_{E} \tilde{\cup} Y_{E}\right) \tilde{\cap} H_{E}\right] \tilde{\cap}\left[b S c l_{\tau}\left(Z_{E} \tilde{\cup} Y_{E}\right) \tilde{\cap} b S c l_{\tau} K_{E}\right] \\
=\left[\left(Z_{E} \tilde{\cup} Y_{E}\right)\right] \tilde{\cap}\left[b S c l_{\tau} K_{E} \tilde{\cap} H_{E}\right]=\tilde{\phi}
\end{gathered}
$$

and

$$
\begin{gathered}
b S c l_{\tau}\left[\left(Z_{E} \tilde{\cup} Y_{E}\right) \tilde{\cap} H_{E}\right] \tilde{\cap}\left[\left(Z_{E} \tilde{\cup} Y_{E}\right) \tilde{\cap} K_{E}\right] \tilde{\subseteq}\left[b S c l_{\tau}\left(Z_{E} \tilde{\cup} Y_{E}\right) \tilde{\cap} b S c l_{\tau} H_{E}\right] \tilde{\cap}\left[\left(Z_{E} \tilde{\cup} Y_{E}\right) \tilde{\cap} K_{E}\right] \\
=\left[\left(Z_{E} \tilde{\cup} Y_{E}\right)\right] \tilde{\cap}\left[b S c l_{\tau} H_{E} \tilde{\cap} K_{E}\right]=\tilde{\phi} .
\end{gathered}
$$

It follows that $\left[Z_{E} \tilde{\cup} Y_{E}\right] \tilde{\cap} K_{E}=Z_{E}$ and $\left[Z_{E} \tilde{\cup} Y_{E}\right] \tilde{\cap} H_{E}=Y_{E}$ are soft b-separated, which is a contradiction. Hence, $Z_{E} \tilde{\cup} Y_{E}$ is soft b-connected relative to $(X, \tau, E)$.

Theorem 4.14. If for all pair of soft points $x_{\alpha}, y_{\beta} \tilde{\in} \tilde{X}$ with $x_{\alpha} \neq y_{\beta}$ there exists a soft b-connected set $Z_{E} \tilde{\subseteq} \tilde{X}$ with $x_{\alpha}, y_{\beta} \tilde{\in} Z_{E}$, then $\tilde{X}$ is soft b-connected.

Proof. Suppose that $\tilde{X}$ is soft b-disconnected. Then, $\tilde{X}=F_{E} \tilde{\cup} G_{E}$, for some $F_{E}, G_{E}$ soft b-separated sets. It follows that, $F_{E} \tilde{\cap} G_{E}=\tilde{\phi}$. So, $\exists x_{\alpha} \tilde{\in} F_{E}$ and $y_{\beta} \tilde{\in} G_{E}$. Since $F_{E} \tilde{\cap} G_{E}=\tilde{\phi}$. Then, $x_{\alpha}, y_{\beta} \tilde{\in} \tilde{X}$ with $x_{\alpha} \neq y_{\beta}$. By hypothesis, there exists a soft b-connected set $Z_{E} \subseteq \tilde{X}$ with $x_{\alpha}, y_{\beta} \tilde{\in} Z_{E}$. Moreover, we have $Z_{E}$ is soft b-connected subset of a soft b-disconnected space. By Theorem 4.7, either $Z_{E} \tilde{\subseteq} F_{E}$ or $Z_{E} \tilde{\subseteq} G_{E}$, and both cases is a contradiction with the hypothesis. This implies that, $\tilde{X}$ is soft b-connected. 
Definition 4.5. A soft set $N_{E}$ is said to be a soft b-neighborhood (briefly, soft b-nbd.) of a soft point $x_{e} \tilde{\in}(X, E)$ if there exists a b-open soft set $U_{E} \tilde{\subseteq} N_{E}$ such that $x_{e} \tilde{\in} U_{E} \tilde{\subseteq} N_{E}$.

Definition 4.6. A soft point $x_{e} \tilde{\in} \tilde{X}$ is called a soft b-limit point of a soft set $F_{E}$ if every soft b-nbd $U_{E}$ of $x_{e}$ contains a point of $F_{E}$ other than $x_{e}$.

Theorem 4.15. Let $F_{E}$ and $G_{E}$ be non-null disjoint soft sets of a soft topological space $(X, \tau, E)$ and $Y_{E}=F_{E} \tilde{\cup} G_{E}$. Then, $F_{E}$ and $G_{E}$ are soft b-separated if and only if each of $F_{E}$ and $G_{E}$ is b-closed soft (b-open soft) with respect to $Y_{E}$.

Proof. Let $F_{E}$ and $G_{E}$ be soft b-separated sets. By Definition 3.1, $F_{E}$ contains no soft b-limit points of $G_{E}$. Then, $G_{E}$ contains all soft b-limit points of $G_{E}$. It follows that, $F_{E} \tilde{\cup} G_{E}$ also contains all soft b-limit points of $G_{E}$ and $G_{E}$ is b-closed soft in $Y_{E}$. Therefore, $G_{E}$ is b-closed soft with respect to $Y_{E}$. Similarly, $F_{E}$ is b-closed soft with respect to $Y_{E}$. The converse is clear from Theorem 4.14.

Theorem 4.16. The soft union of any family of soft b-connected sets having a non-null soft intersection is soft b-connected set.

Proof. Assume that $\left(Z, \tau_{Z}, E\right)=\left(\tilde{\cup}_{j \in J} Z_{j}, \tau_{\tilde{\cup}_{j \in J} Z_{j}}, E\right)$ is soft b-disconnected. Then, $Z_{E}=F_{E} \tilde{\cup} G_{E}$, where $F_{E}, G_{E}$ are soft b-separated sets on $\tau_{Z}$. Since $\tilde{\cap}_{j \in J} Z_{j E} \neq$ $\tilde{\phi}$. Then, $\exists x_{\alpha} \tilde{\in} \tilde{\cap}_{j \in J} Z_{j E}$. It follows that, $x_{\alpha} \tilde{\in} Z_{E}$. So, either $x_{\alpha} \tilde{\in} F_{E}$ or $x_{\alpha} \tilde{\in} G_{E}$. Suppose that $x_{\alpha} \tilde{\in} F_{E}$. Since $x_{\alpha} \tilde{\in} Z_{j E} \forall j \in J$ and $Z_{j E} \tilde{\subseteq} Z_{E}$. Thus, we have $Z_{j E}$ is soft b-connected subset of a soft b-disconnected space. By Theorem 4.7, either $Z_{j E} \tilde{\widetilde{\subseteq}} F_{E}$ or $Z_{j E} \tilde{\subseteq} G_{E} \forall j \in J$. If $Z_{j E} \tilde{\subseteq} F_{E} \forall j \in J$. Then, $Z_{E} \tilde{\subseteq} F_{E}$. This implies that, $G_{E}=\tilde{\phi}$, which is a contradiction. Also, if $Z_{j E} \tilde{\subseteq} G_{E} \forall j \in J$. By a similar way, we can get $F_{E}=\tilde{\phi}$, which is a contradiction. Thus, $\left(Z, \tau_{Z}, E\right)=\left(\tilde{\cup}_{j \in J} Z_{j}, \tau_{\tilde{\cup}_{j \in J} Z_{j}}, E\right)$ is soft b-connected soft subspace of $(X, \tau, E)$.

Proposition 4.1. Let $\left\{\left(Z_{j}, \tau_{Z_{j}}, E\right)\right.$ be a family of soft b-connected subspaces of soft topological space $(X, \tau, E)$ such that one of the members of the family intersects every other members, then $\left(\tilde{\cup}_{j \in J} Z_{j}, \tau_{\tilde{\cup}_{j \in J} Z_{j}}, E\right)$ is soft b-connected.

Proof. Let $\left(Z, \tau_{Z}, E\right)=\left(\tilde{\cup}_{j \in J} Z_{j}, \tau_{\tilde{\cup}_{j \in J} Z_{j}}, E\right)$ and $Z_{j_{o} E} \in\left\{Z_{j E}: j \in J\right\}$ such that $Z_{j_{o} E} \tilde{\cap} Z_{j E} \neq \tilde{\phi} \quad \forall j \in J$. Then, $Z_{j_{o} E} \tilde{\cup} Z_{j E}$ is soft b-connected $\forall j \in J$ from Theorem 4.16. Therefore, the collection $\left\{Z_{j_{o} E} \tilde{\cup} Z_{j E}: j \in J\right\}$ is a collection of a soft b-connected subsets of $\tilde{X}$, which having a non-null soft intersection. Thus, $\left(\tilde{\cup}_{j \in J} Z_{j}, \tau_{\tilde{\cup}_{j \in J} Z_{j}}, E\right)$ is soft b-connected from Theorem 4.16.

Remark 4.2. For a soft topological space $(X, \tau, E)$, the following statements hold:

(1) Every soft $\beta$-connected set is soft b-connected.

(2) Every soft b-connected is soft pre-connected.

(3) Every b-connected is soft semi-connected. 
Definition 4.7. A soft topological space $(X, \tau, E)$ is said to be soft locally bconnected at a soft point $x_{\alpha}$ if every soft b-nbd of the soft point $x_{\alpha}$ contains a soft b-connected open nbd of $x_{\alpha} . \tilde{X}$ is said to be soft locally b-connected if it is soft locally b-connected at each of its soft points.

Theorem 4.17. Every soft b-connected space is a soft locally b-connected space, but the converse is not true in general.

Proof. Suppose that $(X, \tau, E)$ be a soft b-connected. Then, There is no proper b-clopen soft set in $(X, \tau, E)$ from Theorem 4.1. Hence, $\forall x_{\alpha} \tilde{\in} \tilde{X} \exists \tilde{X} \in \tau$ which is soft b-connected set such that $x_{\alpha} \tilde{\in} \tilde{X} \tilde{\subseteq} \tilde{X}$. Therefore, $\tilde{X}$ is soft locally b-connected. On the other hand, the indiscrete soft topological space, is soft locally b-connected but not soft b-connected.

Theorem 4.18. The soft b-component of a soft locally b-connected soft topological space is b-open soft set.

Proof. Let $(X, \tau, E)$ be a soft locally b-connected, $x_{\alpha} \tilde{\in} \tilde{X}$ and $\tilde{S C_{b}}$ be a soft b-component of $\tilde{X}$ w.r.t $x_{\alpha}$. Since $(X, \tau, E)$ is a soft locally b-connected space. Therefore, every b-open soft set containing $x_{\alpha}$ contains a soft b-connected open set $G_{E}$ containing $x_{\alpha}$. But, $\tilde{S C_{b}}$ is the largest soft b-connected set containing $x_{\alpha}$. Hence, $x_{\alpha} \tilde{\in} G_{E} \tilde{\subseteq} \widetilde{S C}$, i.e $\tilde{S C_{b}}$ is a soft b-nbd of $x_{\alpha}$. Thus, $\tilde{S C_{b}}$ is a soft b-nbd of each of its points. This means that, $\tilde{S C_{b}}$ is a b-open soft set.

Theorem 4.19. The property of soft locally b-connectedness is hereditary on $b$ open soft subspaces.

Proof. Suppose that $\left(Z, \tau_{Z}, E\right)$ be a b-open soft subspace of a soft locally b-connected topological space $(X, \tau, E)$ and let $x_{\alpha} \tilde{\in} \tilde{Z}$. Since $\tilde{X}$ is soft locally bconnected. Then, $\exists G_{E} \in \tau$ such that $G_{E}$ is $\tau$-soft b-connected subset of $\tilde{X}$ and $x_{\alpha} \tilde{\in} G_{E} \tilde{\subseteq} \tilde{Z}$. Since $G_{E} \in \tau$ and $G_{E}$ is $\tau$-soft b-connected set. Then, $G_{E}=G_{E} \cap \tilde{\tilde{Z}} \in$ $\tau_{Z}$ and $G_{E} \cap \tilde{Z}$ is $\tau_{Z}$-soft b-connected subset of $\tilde{Z}$ by Theorem 4.6 . So, $\tilde{Z}$ is soft locally b-connected for each $x_{\alpha} \tilde{\in} \tilde{X}$. Hence, $\tilde{Z}$ is soft locally b-connected.

Theorem 4.20. The soft b-components of every b-open soft subspace of a soft locally b-connected soft topological space are b-open soft.

Proof. Suppose that $\left(Y, \tau_{Y}, E\right)$ be a b-open soft subspace of a soft locally bconnected soft topological space $(X, \tau, E)$, let $\tilde{S C_{b}}$ be a soft b-component of $\tilde{Y}$. Since $\tilde{X}$ is soft locally b-connected, then $\tilde{Y}$ is soft locally b-connected by Theorem 4.19. By Theorem 4.18 we get the proof.

Definition 4.8. A soft topological space $(X, \tau, E)$ is said to be soft b-hyperconnected if and only if every pair of non-null proper b-open soft sets $F_{E}, G_{E}$, has a non-null soft intersection, i.e $(X, \tau, E)$ is said to be soft b-hyperconnected if and only if for each $F_{E}, G_{E} \in B O S(X)$, we have $F_{E} \tilde{\cap} G_{E} \neq \tilde{\phi}$. 
Theorem 4.21. Every soft b-hyperconnected soft topological space is soft b-connected.

Proof. Suppose that $(X, \tau, E)$ be a soft b-disconnected soft topological space. Then, there exists a proper b-clopen soft set $F_{E}$. Then, $F_{E}, F_{E}^{c} \in B O S(X)$ such that $F_{E} \tilde{\cap} F_{E}^{c}=\tilde{\phi}$. Hence, $\tilde{X}$ is not soft b-hyperconnected, which is a contradiction. Thus, $(X, \tau, E)$ is soft b-connected.

Remark 4.3. The converse of Theorem 4.21 is not true in general, as shown in the following example.

Example 4.2. Let $X=\left\{h_{1}, h_{2}, h_{3}, h_{4}\right\}, E=\left\{e_{1}, e_{2}\right\}$ and $\tau=\left\{\tilde{X}, \tilde{\phi}, F_{1 E}, F_{2 E}, F_{3 E}\right\}$ where $F_{1 E}, F_{2 E}, F_{3 E}$ are soft sets over $X$ defined as follows:

$F_{1}\left(e_{1}\right)=\left\{h_{1}\right\}, \quad F_{1}\left(e_{2}\right)=\left\{h_{2}\right\}$,

$F_{2}\left(e_{1}\right)=\left\{h_{2}, h_{3}\right\}, \quad F_{2}\left(e_{2}\right)=\left\{h_{1}, h_{3}\right\}$,

$F_{3}\left(e_{1}\right)=\left\{h_{1}, h_{2}, h_{3}\right\}, \quad F_{3}\left(e_{2}\right)=\left\{h_{1}, h_{2}, h_{3}\right\}$.

Then, $\tau$ defines a soft topology on $X$. Hence, the space $(X, \tau, E)$ is soft b-connected but not soft b-hyperconnected.

Definition 4.9. A soft subset $F_{E}$ of a soft topological space $(X, \tau, E)$ is said to be soft b-dense if $b \operatorname{Scl}\left(F_{E}\right)=\tilde{X}$.

Corollary 4.4. A soft topological space $(X, \tau, E)$ is said to be a soft b-hyperconnected if every non-null open soft set $F_{E}$ is soft b-dense.

Theorem 4.22. If $(X, \tau, E)$ is soft b-hyperconnected soft topological space, then $(X, \tau, E)$ is soft hyperconnected.

Proof. Immediate.

Remark 4.4. The converse of Theorem 4.22 is not true in general, as shown in the following example.

Example 4.3. Let $X=\{a, b\}, E=\left\{e_{1}, e_{2}\right\}$ and $\tau=\left\{\tilde{X}, \tilde{\phi}, F_{1 E}, F_{2 E}, F_{3 E}, F_{4 E}, F_{5 E}\right\}$ where $F_{1 E}, F_{2 E}, F_{3 E}, F_{4 E}, F_{5 E}$ are soft sets over $X$ defined as follows:

$F_{1}\left(e_{1}\right)=X, \quad F_{1}\left(e_{2}\right)=\{b\}$,

$F_{2}\left(e_{1}\right)=\{a\}, \quad F_{2}\left(e_{2}\right)=X$,

$F_{3}\left(e_{1}\right)=\{a\}, \quad F_{3}\left(e_{2}\right)=\{b\}$,

$F_{4}\left(e_{1}\right)=\{a\}, \quad F_{4}\left(e_{2}\right)=\{a\}$,

$F_{5}\left(e_{1}\right)=\{a\}, \quad F_{5}\left(e_{2}\right)=\phi$.

Then, $\tau$ defines a soft topology on $X$. Hence, the space $(X, \tau, E)$ is soft hyperconnected, but not soft b-hyperconnected.

Theorem 4.23. . If $(X, \tau, E)$ is soft b-hyperconnected soft topological space, then $(X, \tau, E)$ is connected.

Proof. Immediate by Remark 4.1 and Theorem 4.21. 
Corollary 4.5. The following implications hold from Theorems 4.21, 4.22, 4.23 and [Corollary 3.3, [16]] for a soft topological space $(X, \tau, E)$.

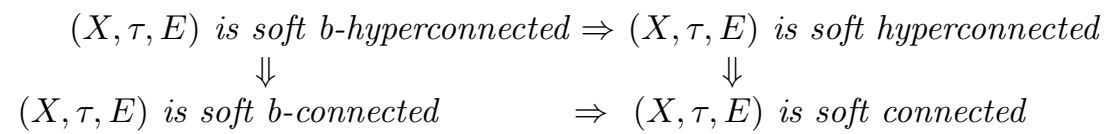

\section{Irresolute soft functions via b-open soft sets}

In this section, we introduce a new type of soft functions called b-irresolute soft function as a generalization to the b-continuous soft functions and obtain some of their properties and characterizations.

Definition 5.1. Let $\left(X, \tau_{1}, A\right)$ and $\left(Y, \tau_{2}, B\right)$ be soft topological spaces and $f_{p u}$ : $S S(X)_{A} \rightarrow S S(Y)_{B}$ be a function. Then, The function $f_{p u}$ is called b-irresolute soft if $f_{p u}^{-1}\left(G_{B}\right) \in B O S(X) \forall G_{B} \in B O S(Y)$.

Theorem 5.1. Every b-irresolute soft function is b-continuous soft.

Proof. Straightforward.

Remark 5.1. The converse of Theorem 5.1 is not true in general, as shown in the following example.

Example 5.1. Let $X=\{a, b, c\}, Y=\{x, y, z\}, A=\left\{e_{1}, e_{2}\right\}$ and $B=\left\{k_{1}, k_{2}\right\}$. Define $u: X \rightarrow Y$ and $p: A \rightarrow B$ as follows:

$u(a)=x, u(b)=z, u(c)=y$,

$p\left(e_{1}\right)=k_{2}, p\left(e_{2}\right)=k_{1}$. Let $\left(X, \tau_{1}, A\right)$ be a soft topological space over $X$ where,

$\tau_{1}=\left\{\tilde{X}, \tilde{\phi}, F_{A}\right\}$, where $F_{A}$ is a soft set over $X$ defined as follows:

$F\left(e_{1}\right)=\{b\}, \quad F\left(e_{2}\right)=\{a\}$.

Let $\left(Y, \tau_{2}, B\right)$ be a soft topological space over $Y$ where,

$\tau_{2}=\left\{\tilde{Y}, \tilde{\phi}, G_{B}\right\}$, where $G_{B}$ is a soft set over $Y$ defined by:

$G\left(k_{1}\right)=\{x\}, \quad G\left(k_{2}\right)=\{x, z\}$.

Let $f_{p u}:\left(X, \tau_{1}, A\right) \rightarrow\left(Y, \tau_{2}, B\right)$ be a soft function. Then, $f_{p u}^{-1}\left(G_{B}\right)=\left\{\left(e_{1},\{a, b\}\right),\left(e_{2},\{a\}\right)\right\}$ is a b-open soft set over $X$. Hence, $f_{p u}$ is a b-continuous soft function. On the other hand, the soft set $H_{B}$, where $H_{B}$ is a soft set over $Y$ defined by:

$H\left(k_{1}\right)=\{y, z\}, \quad G\left(k_{2}\right)=\{x, y\}$.

Then, $H_{B}$ is b-open soft set in $Y$. Also, $f_{p u}^{-1}\left(H_{B}\right)=\left\{\left(e_{1},\{a, c\}\right),\left(e_{2},\{b, c\}\right)\right\}$ is not b-open soft set over $X$. Therefore, $f_{p u}$ is not b-irresolute soft function.

Theorem 5.2. Let $\left(X, \tau_{1}, A\right)$ and $\left(Y, \tau_{2}, B\right)$ be soft topological spaces. Let $u$ : $X \rightarrow Y$ and $p: A \rightarrow B$ be a mappings. Let $f_{p u}: S S(X)_{A} \rightarrow S S(Y)_{B}$ be a function. Then, the following are equivalent:

(1) $f_{p u}$ is b-irresolute soft function. 
Soft Connected Properties and Irresolute Soft Functions Based on -Open Soft Sets 963

(2) $f_{p u}^{-1}\left(H_{B}\right) \in B C S(X) \forall H_{B} \in \tau_{2}^{c}$.

(3) $f_{p u}\left(b S c l_{\tau_{1}}\left(G_{A}\right)\right) \tilde{\subseteq} b S c l_{\tau_{2}}\left(f_{p u}\left(G_{A}\right)\right) \forall\left(G_{A}\right) \in S S(X)_{A}$.

(4) $b S c l_{\tau_{1}}\left(f_{p u}^{-1}\left(H_{B}\right)\right) \tilde{\subseteq} f_{p u}^{-1}\left(b S c l_{\tau_{2}}\left(H_{B}\right)\right) \forall H_{B} \in S S(Y)_{B}$.

(5) $f_{p u}^{-1}\left(\right.$ Sint $\left._{\tau_{2}}\left(H_{B}\right)\right) \tilde{\tilde{\subseteq}} b \operatorname{Sint}_{\tau_{1}}\left(f_{p u}^{-1}\left(H_{B}\right)\right) \forall H_{B} \in S S(Y)_{B}$.

Proof.

$(1) \Rightarrow(2)$ Let $H_{B} \in \tau_{2}^{c}$. Then, $H_{B}^{c} \in \tau_{2}$ and $f_{p u}^{-1}\left(H_{B}^{c}\right) \in B O S(X)$ from Definition 5.1. Since $f_{p u}^{-1}\left(H_{B}^{c}\right)=\left(f_{p u}^{-1}\left(H_{B}\right)\right)^{c}$ from [[30], Theorem 3.14]. Thus, $f_{p u}^{-1}\left(H_{B}\right) \in B C S(X)$.

$(2) \Rightarrow(3)$ Let $G_{A} \in S S(X)_{A}$. Since $G_{A} \tilde{\subseteq} f_{p u}^{-1}\left(f_{p u}\left(G_{A}\right)\right) \tilde{\subseteq} f_{p u}^{-1}\left(b S c l_{\tau_{2}}\left(f_{p u}\left(G_{A}\right)\right)\right) \in$ $B C S(Y)$ from (2) and [[30], Theorem 3.14]. Then, $G_{A} \tilde{\subseteq} b S c l_{\tau_{1}}\left(G_{A}\right) \tilde{\subseteq} f_{p u}^{-1}\left(b S c l_{\tau_{2}}\right.$ $\left.\left(f_{p u}\left(G_{A}\right)\right)\right)$. Hence,

$$
\left.f_{p u}\left(b S c l\left(G_{A}\right)\right) \tilde{\subseteq} f_{p u}\left(f_{p u}^{-1}\left(b S c l_{\tau_{2}}\left(f_{p u}\left(G_{A}\right)\right)\right)\right) \tilde{\subseteq} b S c l_{\tau_{2}}\left(f_{p u}\left(G_{A}\right)\right)\right)
$$

from [[30], Theorem 3.14]. Thus, $f_{p u}\left(b \operatorname{Scl}_{\tau_{1}}\left(G_{A}\right)\right) \tilde{\tilde{\subseteq}} b S c l_{\tau_{2}}\left(f_{p u}\left(G_{A}\right)\right)$.

$(3) \Rightarrow$ (4) Let $H_{B} \in S S(Y)_{B}$ and $G_{A}=f_{p u}^{-1}\left(H_{B}\right)$. Then, $f_{p u}\left(b S c l_{\tau_{1}}\left(f_{p u}^{-1}\left(H_{B}\right)\right)\right) \tilde{\tilde{\subseteq}} b S c l_{\tau_{2}}\left(f_{p u}\left(f_{p u}^{-1}\left(H_{B}\right)\right)\right)$ From (3). Hence,

$$
\begin{aligned}
b S c l_{\tau_{1}}\left(f_{p u}^{-1}\left(H_{B}\right)\right) & \tilde{\subseteq} f_{p u}^{-1}\left(f_{p u}\left(b S c l_{\tau_{1}}\left(f_{p u}^{-1}\left(H_{B}\right)\right)\right)\right) \\
& \tilde{\subseteq} f_{p u}^{-1}\left(b S c l_{\tau_{2}}\left(f_{p u}\left(f_{p u}^{-1}\left(H_{B}\right)\right)\right)\right) \tilde{\subseteq} f_{p u}^{-1}\left(b S c l_{\tau_{2}}\left(H_{B}\right)\right)
\end{aligned}
$$

from [[30], Theorem 3.14]. Thus, $b \operatorname{Scl}_{\tau_{1}}\left(f_{p u}^{-1}\left(H_{B}\right)\right) \tilde{\subseteq} f_{p u}^{-1}\left(b S c l_{\tau_{2}}\left(H_{B}\right)\right)$.

$(4) \Rightarrow(2)$ Let $H_{B}$ be a closed soft set over $Y$. Then,

$$
\begin{aligned}
b S c l_{\tau_{1}}\left(f_{p u}^{-1}\left(H_{B}\right)\right) & \tilde{\subseteq} f_{p u}^{-1}\left(b S c l_{\tau_{2}}\left(H_{B}\right)\right) \\
& =f_{p u}^{-1}\left(H_{B}\right)=f_{p u}^{-1}\left(H_{B}\right) \forall H_{B} \in S S(Y)_{B}
\end{aligned}
$$

from (4), but clearly

$f_{p u}^{-1}\left(H_{B}\right) \tilde{\simeq} b S c l_{\tau_{1}}\left(f_{p u}^{-1}\left(H_{B}\right)\right)$. This means that

$$
f_{p u}^{-1}\left(H_{B}\right)=b S c l_{\tau_{1}}\left(f_{p u}^{-1}\left(H_{B}\right)\right) \in B C S(X) .
$$

$(1) \Rightarrow(5)$ Let $H_{B} \in B O S(Y)$. Then, $f_{p u}^{-1}\left(b \operatorname{Sint}_{\tau_{2}}\left(H_{B}\right)\right) \in B O S(X)$ from (1). Hence, $f_{p u}^{-1}\left(\operatorname{Sint}_{\tau_{2}}\left(H_{B}\right)\right)=b_{\operatorname{Sint}_{\tau_{1}}}\left(f_{p u}^{-1} b \operatorname{Sint}_{\tau_{2}}\left(H_{B}\right)\right) \tilde{\subseteq} b \operatorname{Sint}_{\tau_{1}}\left(f_{p u}^{-1}\left(H_{B}\right)\right)$. Thus, $f_{p u}^{-1}\left(\operatorname{Sint}_{\tau_{2}}\left(H_{B}\right)\right) \tilde{\subseteq} b \operatorname{Sint}_{\tau_{1}}\left(f_{p u}^{-1}\left(H_{B}\right)\right)$.

$(5) \Rightarrow(1)$ Let $H_{B} \in B O S(Y)$. Then, $\operatorname{SSint}_{\tau_{2}}\left(H_{B}\right)=H_{B}$ and

$$
f_{p u}^{-1}\left(\operatorname{Sint}_{\tau_{2}}\left(H_{B}\right)\right)=f_{p u}^{-1}\left(\left(H_{B}\right)\right) \tilde{\subseteq} b \operatorname{Sint}_{\tau_{1}}\left(f_{p u}^{-1}\left(H_{B}\right)\right)
$$


from (5). But, we have bint $_{\tau_{1}}\left(f_{p u}^{-1}\left(H_{B}\right)\right) \tilde{\subseteq} f_{p u}^{-1}\left(H_{B}\right)$. This means that,

$$
\operatorname{bint}_{\tau_{1}}\left(f_{p u}^{-1}\left(H_{B}\right)\right)=f_{p u}^{-1}\left(H_{B}\right) \in \operatorname{BOS}(X) .
$$

Thus, $f_{p u}$ is b-irresolute soft function.

Theorem 5.3. Let $\left(X_{1}, \tau_{1}, A\right)$ and $\left(X_{2}, \tau_{1}, B\right)$ be soft topological spaces, $f_{p u}$ : $\left(X_{1}, \tau_{1}, A\right) \rightarrow\left(X_{2}, \tau_{2}, B,\right)$ be a surjective b-irresolute soft function and $F_{B}, G_{B}$ are soft b-separated sets in $X_{2}$, then $f_{p u}^{-1}\left(F_{B}\right), f_{p u}^{-1}\left(G_{B}\right)$ are soft b-separated sets in $X_{1}$.

Proof. Let $F_{B}, G_{B}$ be soft b-separated sets in $X_{2}$. Then, $F_{B} \tilde{\cap} b S c l\left(G_{B}\right)=$ $\tilde{\phi}_{B}$ and $b S c l\left(F_{B}\right) \tilde{\cap} G_{B}=\tilde{\phi}_{B}$. Since $b S c l_{\tau_{1}}\left(f_{p u}^{-1}\left(H_{B}\right)\right) \tilde{\subseteq} f_{p u}^{-1}\left(b S c l_{\tau_{2}}\left(H_{B}\right)\right) \forall H_{B} \in$ $S S\left(X_{2}\right)_{B}$ from Theorem $5.2(4)$. It follows that

$$
\begin{aligned}
f_{p u}^{-1}\left(G_{B}\right) \tilde{\cap} b S c l_{\tau_{1}}\left(f_{p u}^{-1}\left(H_{B}\right)\right) & \tilde{\subseteq} f_{p u}^{-1}\left(G_{B}\right) \tilde{\cap} f_{p u}^{-1}\left(b S c l_{\tau_{2}}\left(H_{B}\right)\right) \\
& =f_{p u}^{-1}\left[G_{B} \tilde{\cap} b S c l_{\tau_{2}}\left(H_{B}\right)\right]=f_{p u}^{-1}\left(\tilde{\phi}_{B}\right)=\tilde{\phi}_{A} .
\end{aligned}
$$

By a similar way, we have $f_{p u}^{-1}\left(H_{B}\right) \tilde{\cap} b S c l_{\tau_{1}}\left(f_{p u}^{-1}\left(G_{B}\right)\right)=\tilde{\phi}_{A}$. Therefore, $f_{p u}^{-1}\left(F_{B}\right), f_{p u}^{-1}\left(G_{B}\right)$ are soft b-separated sets in $X_{1}$.

Theorem 5.4. Let $\left(X_{1}, \tau_{1}, A\right)$ and $\left(X_{2}, \tau_{1}, B\right)$ be soft topological spaces and $f_{p u}$ : $\left(X_{1}, \tau_{1}, A\right) \rightarrow\left(X_{2}, \tau_{2}, B,\right)$ be a bijective b-irresolute soft function. If $G_{A}$ is soft $b$-connected in $X_{1}$, then $f_{p u}\left(G_{A}\right)$ is soft b-connected in $X_{2}$.

Proof. Suppose that $f_{p u}\left(G_{A}\right)$ is not soft b-connected in $X_{2}$. Then, $f_{p u}\left(G_{A}\right)=$ $M_{B} \tilde{\cup} N_{B}$ for some soft b-separated sets $M_{B}, N_{B}$ of $f_{p u}\left(G_{A}\right)$ in $X_{2}$ from Theorem 4.1. By Theorem 5.3, $f_{p u}^{-1}\left(M_{B}\right)$ and $f_{p u}^{-1}\left(N_{B}\right)$ are soft b-separated in $X_{1}$. Since $f_{p u}$ is bijective soft function. So, $G_{A}=f_{p u}^{-1}\left(f_{p u}\left(G_{A}\right)\right)=f_{p u}^{-1}\left(M_{B}\right) \tilde{\cup} f_{p u}^{-1}\left(N_{B}\right)$. It follows that, $G_{A}$ is not soft b-connected in $X_{1}$, which is a contradiction. Thus, $f_{p u}\left(G_{A}\right)$ is soft b-connected in $X_{2}$.

Corollary 5.1. Let $\left(X_{1}, \tau_{1}, A\right)$ and $\left(X_{2}, \tau_{1}, B\right)$ be soft topological spaces and $f_{p u}$ : $\left(X_{1}, \tau_{1}, A\right) \rightarrow\left(X_{2}, \tau_{2}, B,\right)$ be a surjective b-irresolute soft function. If $X_{1}$ is soft $b$-connected space, then so $X_{2}$.

Proof. Follows from Theorem 5.4.

Theorem 5.5. Let $\left(X_{1}, \tau_{1}, A\right)$ and $\left(X_{2}, \tau_{1}, B\right)$ be soft topological spaces and $f_{p u}$ : $\left(X_{1}, \tau_{1}, A\right) \rightarrow\left(X_{2}, \tau_{2}, B,\right)$ be a surjective $b$-continuous soft function. If $G_{A}$ is soft $b$-connected in $X_{1}$, then $f_{p u}\left(G_{A}\right)$ is soft connected in $X_{2}$.

Proof. The proof is similar to the proof of Theorem 5.4.

Corollary 5.2. Let $\left(X_{1}, \tau_{1}, A\right)$ and $\left(X_{2}, \tau_{1}, B\right)$ be soft topological spaces and $f_{p u}$ : $\left(X_{1}, \tau_{1}, A\right) \rightarrow\left(X_{2}, \tau_{2}, B,\right)$ be a surjective b-continuous soft function. If $X_{1}$ is soft $b$-connected space, then $X_{2}$ is soft connected.

Proof. Follows from Theorem 5.5. 


\section{Conclusion}

In the present paper, we introduce and study the notion of connectedness to soft topological spaces by using the notion of b-open soft sets. We study the notions of soft b-connected sets, soft b-separated sets in soft topological spaces and have established several interesting properties. Further, we introduce the notion of birresolute soft functions as a generalization to the b-continuous soft function and study their properties in detail. Finally, we show that the surjective b-irresolute soft image of soft b-connected space is also soft b-connected. Our next step is to generalize these notions by using the soft ideal notions [17].

\section{Acknowledgements}

The author expresses his sincere thanks to the reviewers for their valuable suggestions. The author is also thankful to the editors-in-chief and managing editors for their important comments which helped to improve the presentation of the paper.

\section{R E F E R E N C ES}

1. A. M. AbD El-Latif and Serkan Karatas: Supra b-open soft sets and supra b-soft continuity on soft topological spaces. Journal of Mathematics and Computer Applications Research, 2 (1) (2015), 1-18.

2. A. M. AbD El-LATIF: Soft connectedness and irresoluteness via $\beta$-open soft sets. Honam Mathematical Journal. Accepted.

3. A. M. AbD EL-LATi: Soft supra strongly generalized closed sets. Journal of Intelligent \& Fuzzy Systems, vol. Preprint, no. Preprint (2016), pp. 1-7.

4. B. Ahmad and A. Kharal: Mappings on fuzzy soft classes. Adv. Fuzzy Syst. 2009, Art. ID 407890, 6 pp.

5. B. Ahmad and A. Kharal: Mappings on soft classes. New Math. Nat. Comput., 7 (3) (2011), 471-481.

6. M. Akdag and A. Ozkan: Soft b-open sets and soft b-continuous functions. Math. Sci. (2014) 8:124, , DOI 10.1007/s40096-014-0124-7.

7. M. I. Ali, F. Feng, X. Liu, W. K. Min and M. Shabir: On some new operations in soft set theory. Comput. Math. Appl., 57 (2009), 1547-1553.

8. Bin Chen: Some Local Properties of Soft Semi-Open Sets. Hindawi Publishing Corporation, 2013 (1-6).

9. N. agman and S. Enginoglu: Soft set theory and uni-Fint decision making. European Journal of Operational Research, 207 (2010), 848-855.

10. S. A. El-Sheikh and A. M. AbD El-LATiF: Characterization of b-open soft sets in soft topological spaces. Journal of New Theory, 2 (2015), 8-18.

11. S. A. El-Sheikh and A. M. AbD El-LATIF: Decompositions of some types of supra soft sets and soft continuity. International Journal of Mathematics Trends and Technology, 9 (1) (2014), 37-56. 
12. S. A. El-Sheikh, Rodyna A. Hosny and A. M. Abd El-latif: Characterizations of b-soft separation axioms in soft topological spaces. Inf. Sci. Lett., 4 (3) (2015), 125-133.

13. S. Hussain and B. Ahmad: Some properties of soft topological spaces. Comput. Math. Appl., 62 (2011), 4058-4067.

14. A. Kandil, O. A. E. Tantawy, S. A. El-Sheikh and A. M. Abd El-latif: $\gamma$-operation and decompositions of some forms of soft continuity in soft topological spaces. Ann. Fuzzy Math. Inform., 7 (2) (2014), 181-196.

15. A. Kandil, O. A. E. Tantawy, S. A. El-Sheikh and A. M. Abd El-latif: $\gamma$-operation and decompositions of some forms of soft continuity of soft topological spaces via soft ideal. Ann. Fuzzy Math. Inform., 9 (3) (2015), 385-402.

16. A. Kandil, O. A. E. Tantawy, S. A. El-Sheikh and A. M. Abd El-latif: Soft connectedness via soft ideals. Journal of New Results in Science, 4 (2014), 90-108.

17. A. Kandil, O. A. E. Tantawy, S. A. El-Sheikh and A. M. Abd El-latif: Soft ideal theory, Soft local function and generated soft topological spaces. Appl. Math. Inf. Sci., 8 (4) (2014), 1595-1603.

18. A. Kandil, O. A. E. Tantawy, S. A. El-Sheikh and A. M. AbD El-latif: Soft regularity and normality based on semi open soft sets and soft ideals. Appl. Math. Inf. Sci. Lett., 3 (2) (2015), 47-55.

19. A. Kandil, O. A. E. Tantawy, S. A. El-Sheikh and A. M. Abd El-latif: Soft semi compactness via soft ideals .Appl. Math. Inf. Sci., 8 (5) (2014), 22972306.

20. A. Kandil, O. A. E. Tantawy, S. A. El-Sheikh and A. M. Abd El-latif: Soft semi (quasi) Hausdorff spaces via soft ideals. South Asian J. Math., 4 (6) (2014), 265-284.

21. A. Kandil, O. A. E. Tantawy, S. A. El-Sheikh and A. M. Abd El-latif: Soft semi separation axioms and irresolute soft functions. Ann. Fuzzy Math. Inform., 8 (2) (2014), 305-318.

22. A. Kandil, O. A. E. Tantawy, S. A. El-Sheikh and A. M. Abd El-latif: Supra generalized closed soft sets with respect to an soft ideal in supra soft topological spaces. Appl. Math. Inf. Sci., 8 (4) (2014), 1731-1740.

23. F. Lin: Soft connected spaces and soft paracompact spaces. International Journal of Mathematical Science and Engineering, 7 (2) (2013), 1-7.

24. P. K. Maji,R. Biswas and A. R. Roy: Soft set theory. Comput. Math. Appl., 45 (2003), 555-562.

25. D. Molodtsov: Soft set theory-firs tresults. Comput. Math. Appl., 37 (1999), 19-31.

26. D. Molodtsov, V. Y. Leonov and D. V. Kovkov: Soft sets technique and its application. Nechetkie Sistemy i Myagkie Vychisleniya, 1 (1) (2006), 8-39.

27. D. Pei and D. Miao: From soft sets to information systems, in: X. Hu, Q. Liu, A. Skowron, T. Y. Lin, R. R. Yager, B. Zhang (Eds.). Proceedings of Granular Computing, in: IEEE, vol.2, 2005, pp. 617-621.

28. Rodyna A. Hosny: Properties of soft b-open sets. SYLWAN., 159 (4) (2014), $34-49$. 
Soft Connected Properties and Irresolute Soft Functions Based on -Open Soft Sets 967

29. M. ShaBiR and M. NAZ: On soft topological spaces. Comput. Math. Appl., 61 (2011), 1786-1799.

30. I. Zorlutuna, M. Akdag, W.K. Min and S. Atmaca: Remarks on soft topological spaces. Ann. Fuzzy Math. Inform., 3 (2) (2012), 171-185.

Alaa Mohamed Abd El-latif

Ain Shams University

Faculty of Education

Department of Mathematics

Roxy, 11341, Cairo, Egypt

alaa_8560@yahoo.com 